\title{
Discussion on Western Region Music in Northern Zhou Dynasty
}

\author{
Xing Tang \\ School of Literature \\ Northwest Minzu University \\ Lan zhou, China
}

\begin{abstract}
The Northern Zhou Dynasty (induding Western Wei Dynasty), as a dynasty that serve as a link between past and future, should have its place in the cultural history. In the Northern Zhou Dynasty, various cultures in Western Regions were closely interwoven. As one of the important representatives, the music presented a wide range of sources with a large number of complicated compositions. These Western music has unique style but also coexistence which has very close relationship with Northern Zhou's music, so it has great influence to the music of Northern Zhou Dynasty and even the music of future generations.
\end{abstract}

Keywords—Northern Zhou Dynasty; Western Regions; music genre; mu sical instrument

\section{INTRODUCTION}

The Western Regions are a geographical concept with historic and political change. The geographical scope of the Western Regions during the Northem and Southern Dynasties was roughly equivalent to the whole part of Xinjiang and a part of Central Asia [1]. During the Northern Zhou Dynasty, the Western countries mainly included Shanshan, Gaochang, Yanqi, Qiuci, Yutian, Shule, Zhada, Sute, as well as the "Oghuz"'s city-states such like Kangguo, Anguo, Caoguo, Miguo, Heguo, Shiguo, Shiguo etc. The coexistence of various countries and the multi-ethnic blending in the Western Regions have profoundly affected the development of local culture. Taking music as an example, the Western region has the characteristics of wide range of sources, large amount, complicated composition and integration.

\section{MUSIC SOURCE OFTHE WESTERN REGIONS IN NORTHERN ZHOU DYNASTY}

With the opening of the "Silk Road" in Han Dynasty, the source channels and genres of music in the western region gradually became stereotyped from the Han Dynasties to the Wei and Jin Dynasties, sixteen kingdoms, to the Northern Wei Dynasty and the Northern Zhou Dynasty.

Western music from the source can be divided into local music and foreign music.

Local music includes ancient music, later music and current music. Ancient music is the Western music before the

Fund Project: Basic research funding special fund-funded graduate programs of Northwest Minzu University (project number: Yxm2017002.
Western and Eastern Han Dynasty, which include folk witch music dance, welcome God music, send God music, Xiangge and other songs; Later music is the Western music after the Han Dynasty and before Western Wei Dynasty, which include game music, ritual music, custom music and so on; Current music is the Western music in Northern Zhou Dynasty, due to the large number of countries in the Western Regions and the constant attacking and frequent destruction of the Western Regions, so current music in fact refers to the remnants and integration of the music of different countries in different historical periods in the Western Regions, which include Shengming music, Tuozhi music and so on.

Foreign music can be broadly divided into Foreign West music, Foreign South music, Foreign North music and Foreign East four categories. Foreign West music mainly refers to the music of West Asia, North Africa and Europe at that time, such as the Muhu music from Persia. Foreign South music mainly refers to the music of South Asia and Southeast Asia at that time, such as Sha Shijiang from Thindu, dance music Suhe Xiang and Tianqu; Foreign North music mainly refers to the music of North Asia area at that time, such as the dance music Damo Zhi from the Turks; Foreign East music mainly refers to the music from Central Plains at that time, such as the Qingle, Xianghe dance music from the Han and Jin Dynasties and Northern and Southern Dynasties.

These music from different sources if study from the sound structure including instrumental, vocal and dance music. There are ensembles and solo performances for two kinds of instrumental music performance; As for vocal performances it include solo, duet, lead singing and helmets performance types; For dance music it has solo dance music, double dance music, triple dance music and group dance music, singles and songcycles. Study from the lyrics structure, there are section songs, verse and chorus songs, Huantou songs, Lian songs and so on. Study from the theme content, there are love songs, working songs, customs songs, satirical songs and so on. Study from the genre, there are lyric songs, narrative songs, allegory songs, long songs and short songs. Study from the style, they are normally multi-paced and bright with high pitched tone, warm and imaginative, lively and vivid. Study from the phonetic tone, they are mainly ethnic minorities, also with a few Han tone and Sanskrit tone. 


\section{THE GENRE OF THE WESTERN REGIONS MUSIC IN NORT HERN ZHOU DYNAST Y}

Western Regions music from the music genre have Qiuci music, Shule music, Kangguo music, Anguo music, Gaochang music, Tianzhu music, Yutian music, Yueban music and so on.

According to the record from "Sui Shu. Yin Yue Zhi" that Qiuci music's "Song music has Shan Shan Mo Ni, Jie music has Po Jia Er, Dance music has Xiao Tian and Shu Le Yan" [2] (P379); Shule music's " Song music has Kangli sirang yue, Dance music has Yuan fu, Jie music has Yan qu"[2] (P380); Kang Guo music's " Song music has Jidian Nonghe Zheng, Dance music has Helan Bobi Shil, Moxi Bodi, Nonghui Bobi Shi, Qian Badi Huid i four songs "[2] (P379); An Guo music's " Song music has Fusa Danshi, Dance music has Moxi, Jie music has Ju He Zhi"[2] (P380)," Wei Shu · Tai zu Ji "also recorded that Song music has Ge zhi xi, Dance music has Wu Zhi Xi; Gao Chang music has" Sheng Ming music "[2] (P379); Tianzhu music's" Songs music has Sha Shi Jiang, Dance music has Tian Qu " [2] (P379). According to "Wei Shu . Yue Zhi" mentioned when Zhang Qian from the Western Han Dynasty mission to the West Regions, he got Mohe Doule song, which is derived from Tianzhu after textual research, so that we could know the songs introduced to Western Regions from Tianzhu should include Mohe Doule song; Yu Tian music has Yu Tian Fo song; Yueban music mostly are drums dance music, "Wei Shu.Yue Zhi" mentioned that in Northern Wei Dynasty "The Drums dance music was introduced into the music department" [3] (P2828).

According to the preliminary statistics, there are 23 music genres in total, of which there are at least 5 genres of "Song music" mainly used for singing, at least 3 genres of "Jie music" used to supplement the lack of music meaning, at least 11 genres of "Dance music" used for dance performance.

Besides that, according to the research of experts such as Gao Nan, Ku Lang, Sha Wan, Le Wei, Tanabe, Bo Xihe and others, the "Tara" rhythms and tempo system in Tianzhu music and Sa, Re, Gs, Ma, Pa Da, Ni "Svara-Rag" style seven-scale soundtracks were also introduced into the Western Regions. The Western Regions local music which represented by Quci music have created the "Five Psalms and Seven Tones" sound system after the leaning and absorb other music. This sound system promoted the music development of the entire Western Regions area. It also had a profound impact on the musical standards, sound systems and sound arrangements of contemporary and later musical instruments.

\section{THE GENERAL SIT UATION OF WESTERN REGIONS MUSICAL INST RUMENTS IN NORT HERN ZHOU DYNASTY AND LOCAL MUSICAL INST RUMENTS}

There are plenty amount and types of Western Regions musical instruments, so you can see how prosperous of local musical culture at that time. However, based on preliminary statistics, there are only a few types of local musical instruments, identified local musical instruments are only about $29 \%$ of the total number, so the development of Western Region music seemed more depend on the promotion of foreign music.
During the Northern Zhou Dynasty, the musical instruments used in the Western Regions at least include Xun, Sheng, Hu guan, flute/Hu chi, bamboo flute, Tatar flute, India flute, Chi, Xiao, Yue, Huang, Guan, Bi li, Small Bi li, Bi li with peach tree skin, Double Bi li, Horn, Copper Horn, Long horn, Horizontal blowing horn, Jia, Suona, Bei, Luo, Kong hou, Shu Konghou, Konghou with Phoenix head, Ruanxian, Wuxian, Curve-neck Pipa, Qin, Se, Yu, Drums, Small drums, Dabo, Zheng Drums, He Drums, Zha Drums, Dala Drums, Jilou Drums, Qi Drums, Yan Drums/Dan Drums, Kai Drums, Houti Drums, Jie Drums, Copper Drums, Maoyuan Drums, Dutan Drums, Waist Drums/Zhang Drums, Jia Drums, Bell / Peng Qian, Jian Zhong,Copper cymbals, Nao, total 54 types.

In addition, there may also have large $\mathrm{Bi}$ li, vertical s mall Bi li, Zheng, Tan Zheng, Chou Zheng, Tao, Jian Drums, Qi Drums, Ben drums, Xuan drums, Jie drums, Hu drums, Bing drums, Bian drums, Bofu, Zhu, Yu, Clapper, Zhu, Sand gongs / Copper gongs, Copper bowl, Fang Xiang, Xing / Peng ling and other musical instruments, with the previous 54, total about 77 types.

Identified Western Regions local musical instruments at least have 15 types.

There are at least 5 kinds of wind instruments. Qiang flute, which is flute, is a horizontal blowing flute, the Han Dynasty Ma Rong's "Chang Di Fu" mentioned "the current flute came from Qiang". Qiang horn, also known as $\mathrm{Hu}$ horn, that is straight blowing horn. Double horn, which is horn, according to "Wen Xian Tong Kao" said: "double horn also known as Changming horn there is no record about it". Copper horn, Xiao Youmei's "Ancient Chinese Musical Instrument research" said that it was produced in Turkey that is the area of Xinjiang [4] (P121), seems to be evolved from the early animal horn musical instruments of Qiang. Horizontal blowing horn, is a modified form of the early horn. "Shuo Wen Jie Zi" explained "horn" as Qiang's horn, so all these above are Qiang musical instruments. "Han Shu. Western Regions Biography" recorded that the south of Qiang connected with Ruoqiang, Xiuxun was on the south of Qiang and Dayuezhi was in the west of Qiang. So Qiang was also called Western Qiang, is an ancient nation in northwestern China and Central Asia [5] (P107). Therefore, these musical instruments can be regarded as Western Regions local musical instruments.

There are at least 10 kinds of percussion instruments. Dabo, which is tambourines, is still very common in Xinjiang music and dance; Zheng drums, He drums, Zha drums, Dala drums, Jilou drums, Qi drums, Yan drums, Kai drums, Houti drums are all from Turkestan, now Xinjiang region [4] (P103-105) according to Xiao Youmei's research. The author said from here, do not make detailed introduction.

\section{THE FOREIGN MUSICAL INST RUMENTS FROM WESTERN REGIONS MUSIC IN NORT HERN ZHOU DYNASTY}

According to the previous criteria for allocating foreign music, there are at least 35 identified foreign musical instruments in the Western Regions music, among which the Foreign East musical instruments has the largest amount, Foreign south musical instruments are the second, accounting 
for $48 \%$ and $31 \%$ of the total number of external musical instruments respectively.

The identified Foreign East musical instrument has 17 kinds. Sheng, Huang, Qin, Se, Yu, Lun, Zhong, Drums, Jian Zhong, Wan, these Central Plains musical instruments were believed by Zhou Jingbao that they have spread to the Western Regions during the Pre-Qin period. Among them, Sheng and Huang have been mastered by the ancient ethnic groups in the Western Regions [6] (P36, 48, 76), as for Qin, according to "Han Shu. Western Regions Biography", it is recorded that when Princess Xijun and Princess Jieyou married to Wusun, they brought this musical instrument, but the mark of real mastery is the king of Qiuci, Jiangbin couple went to Changan to learn how to play Qin in Emperor Han Xuan period. Konghou, mainly refers to Wo Konghou, according to the literature records it was created by musicians' in Han Dynasty, the tomb of Zhang Lichen from Han Dynasty which located in Xinjiang Astana area has unearthed silk paintings with the image of musicians held Wo Konghou. Ruan Xian, also called Ruan, mostly refer to Qin Pipa . Xun, earliest seen as the pottery Xun without sound hold, pottery Xun with 1 sound hold and pottery Xun with 2 sound holes which unearthed in Shanxi Wanquanjing village Neolithic sites, and pottery Xun with 1 sound hold unearthed in Zhejiang Hemudu cultural relics, in 1995 in Xinjiang Moyu County Mulabate sites unearthed a pottery Xun with 3 sound holds, and it was identified as the cultural relic from 5-10 century. Chi, also known as $\mathrm{Ba}$, in "Shijing. Xiaya . Herensi" has the "Mr. Zhong blow Chi " sentence, Chen Yang's "Yue Shu" recorded "Chi is a kind of musical instrument, it is a flute with bottom", Mr Yang Yinliu classified it as an ancient musical instrument of Central Plains [7] (P69). Nao, earliest found in Longshan site from Shaanxi province Chang'an county Kesheng village which unearthed pottery Nao, in Shang Dynasty appeared copper nao and divided into big and small two kinds, the big kind named Yong, Zeng Houyi from small kind named Nao, and even appeared Nao set [8] (P5). Bongo, the Tomb of Zeng Houyi from the Warring States unearthed such instruments, normally seen in the Han Dynasty's Shuochang figurines. Pai xiao, that is Feng xiao, normally refers to Duan xiao, the tomb of Zeng Houyi from Hubei Suixiang County unearthed Pai xiao from early Warring States period. "Gan zhu" mentioned "Duan xiao with twenty-one holds is a military musical instrument", "Wen Xian Tong Kao "recorded "Xiao with twenty-one holes, this xiao has seven tones and 3 times one scales which used by Qiuc..

In addition, musical instruments that may be introduced to the Central Plains are: Qing, Zheng, Tao, Bofu, Zhu/Kong, $\mathrm{Yu} / \mathrm{J}$ ie, Paiban, Jian drums/Ying drums/Ying drums/Zhi drums/Big drums, Pao drums, Ben drums, Xuan drums, Zhu, Jie drums and so on.

The identified Foreign South musical instrument has 11 kinds. For the Kong Hou with phoenix head, the "Sui Shu. Yin Yue Zhi", "Jiu Tang Shu. Yin Yue Zhi", "Xin Tang Shu. Li Yue Zhi", and "Tong Dian", "Tang Liu Dian" all recorded "Kong Hou with phoenix head, every Tianzhu musicians has one"; the "Wen Xian Tong Kao" also recorded that "phoenix head was first came from Tianzhu musicians, it has curved neck and phoenix shape. The Phoenix head Kong Hou fro $\mathrm{m} \mathrm{Fu}$
Lou (ancient Vietnam), Gaochang and other countries are very exquisite", in Xinjiang Kizil Buddha Cave painted this instrument. The five-stringed pipa, Chang Renxia consider it came from ancient India, is found in carvings of the Amravati (about 170 AD) in Central India, and is the same shape with the musical instrument seen in Chusi, but it was early before Qiuci. Ans they were also found in the carvings of Janta (300750 BC) in western India [9] (P15). Bei, is Fan Bei; Lo has Yu Luo and Fa Luo. Indian flute, Shen Fuwei believe that Indians during the Rig Vedia period has begun to play flute, Indian flute may have connection with Qiang flute [10] (P74). Copper drums, Dutan drums, Maoyuan drums are all came from India according to Xiao Youmei's research[4] (P105). Jie drum, which is Zhang drum, comes from Indian according to the research of Lin Qiansan textual research [11]. There are paintings of this kind of musical instrument in both India Sanehi tower relief sculptures and Ajanta murals. Bo, there is also copper bo, which is copper plate, introduced at about AD 350 along with Tianzhu music [12] (P90).

Identified Foreign North musical instrument are two kinds. $\mathrm{Jia}$, which is Hu Jia, Chui Bian, one of them was unearthed in Xinjiang Altay in 1985 with 3 holes, made of wood. Heng Chui is also known as flute [13].

The identified Foreign West musical instrument has 5 kinds. Shu Konghou, common known as Bo Konghou, $t$ is generally believed that came from Persia, some scholars believe it comes from Egypt [14] (P12), Cave 23 of Ghazar Kajaha Cave in Xinjiang, and Cave 48 of Bozi Crick all painted with this musical instrument. Curve neck Pipa, mostly refers to Quici Pipa, four strings, according to Kishibe's "Musical Culture of Silk Road" [15] and Lin Qiansan's "Research of Yan Yue music of Sui and Tang Dynasty" [11] ,it came from West Asia. Bi li, also called Guanzi, Lu guan, Chang Ren xia believe that it came from ancient Iran area [9] (P20), Cave 46 of Xinjiang Kunmu Tula Buddha cave has paintings with this musical instrument; Flute, formerly known as Zhu, which is the clarinet, in Tang Dynasty called is as Chi $\mathrm{Ba}$, after Tang and Song Dynasty it was called as Xiao or Dong Xiao, according to TTanabe Chinchon's textual research, it came from West Asia [16]; Suona, also known as Suonai, Suernai, textual research approved it originated in Persia and the Arab region [12] (P75), Cave 38 of Xinjiang Kizil Cave Temple (about AD 256-420) has mural paintings with this musical instrument.

\section{CONCLUSION}

To sum up, the Western Regions as an important geographical area for cultural exchange between East and West, the western Regions music in Northern Zhou Dynasty has shown a fusion trend. Local and foreign music interact with each other and coexist harmoniously. The re-integrated Western Regions music showed its unique musical appeal, full of vitality, and was in the dominate position in North Zhou dynasty's music. After that this music pattern which dominated by the Western Regions Music continued from Northern Zhou Dynasty all the way to Sui and Tang Dynasties, which has a profound impact on the music and music literature of later generations.. 


\section{REFERENCES}

[1] Tian Weijiang. The concept of "Western Regions" and its connotation [J]. Western Regions Research, 1998 (4).

[2] (Tang) Wei Zheng. Sui Shu [M]. Zhonghua Book Company, 1973.

[3] (Northern Qi) Wei Shou. Wei Shu [M]. Zhonghua Book Company, 1974.

[4] Xiao Youmei. Research of Ancient Chinese instrument [M]. Jilin Publishing Group Co., Ltd., 2010.

[5] Jin Qiu. Silk Road Music and Dance Art Research [M]. Xinjiang People's Publishing Company, 2009.

[6] Zhou Jingbao. Music culture of the Silk Road [M]. Xinjiang People's Publishing Company, 1988.

[7] Yang Yinliu. History of Chinese music [M]. Shanghai Wan Ye bookstore, 1952.

[8] Wu Zhao. Ancient Chinese musical instruments [M]. Cultural Relics Publishing Company, 1983.

[9] Chang Renxia. East expansion of Western Regions music art between Han and Tang Dynasties [J]. Music Research, 1980 (4).

[10] Shen Fuwei. History of Chinese and Western cultural exchanges [M]. ShanghaiPeople's Publishing Company, 1985.

[11] (JP) Lin Qiansan. Research of East Asian musical instruments [M]. Translated by Qian Daosun.People's Music Publishing Company, 1962.

[12] Zang Yibing. History of Chinese Music (Revised Edition) [M]. Wuhan University Press, 2006.

[13] Yusaiyin · Klimu. Research of Huon music [J]. Xinjiang Art, 1989 (3).

[14] Song Bonian, Li Qiang. Music Research of Silk Road [M]. Xinjiang People's Publishing House, 2009.

[15] (JP) Kishibe. Musical Culture of Silk Road [M]. Translated by Wang Yaohua. People's Music Publishing Company, 1988.

[16] Tanabe Chinchon. History of Chinese music [M]. Translated by Chen Qingquan. The Commercial Press, 1937. 